Europhys. Lett., 8 (2), pp. 111-116 (1989)

\title{
Directed Polymers on Disordered Hierarchical Lattices.
}

\author{
B. Derrida and R. B. Griffiths $\left(^{*}\right)$ \\ Service de Physique Théorique (**) de Saclay - F-91191 Gif-sur-Yvette Cedex, France
}

(received 17 October 1988; accepted in final form 9 November 1988)

PACS. 02.50 - Probability theory, stochastic processes, and statistics.

PACS. 05.20 - Statistical mechanics.

\begin{abstract}
We study the distribution of ground-state energies of directed polymers on disordered hierarchical lattices. The problem can be reduced to finding the stable distribution when one combines random variables in a nonlinear way (for example, $e=\min \left(e_{1}+e_{2}, e_{3}+e_{4}\right)$ ). The ground-state energy fluctuations of a polymer of length $L$ increase like $L^{\omega}$. We calculate $\omega$ numerically for a whole family of hierarchical lattices. In a limiting case, we present a perturbation theory for the ground-state energy and the exponent $\omega$, which agrees well with the numerical results.
\end{abstract}

Polymers in random media have been a controversial subject for some time [1-4]. The combined effects of excluded volume and randomness are still not fully understood, and the upper critical dimension and the critical exponents remain unknown. There has recently been important progress in the case of directed polymers: in two dimensions the critical exponents are known exactly [5-8], and the exact solution of the "mean field" limit of a polymer on a tree shows several features in common with mean-field spin glasses [9].

It would be interesting to extend these «mean field" results to models of a finitedimensional lattice, but that is a difficult problem. In the present paper we consider some properties of directed polymers on random hierarchical lattices [10] which can be thought of as intermediate between mean-field and finite-dimensional systems [11], or as providing a (crude) renormalization group approximation to the latter. Here we will only consider the zero-temperature limit or ground state; finite-temperature studies are among some of the possible generalizations mentioned in the conclusion. At zero temperature, the directed polymer reduces to an optimization problem which is to find the path of lowest energy in a random landscape [6].

The hierarchical lattices $[10,11]$ we shall consider are constructed by an iterative rule, indicated in fig. 1a) for the «diamond» lattice. The first generation consists of one bond and two sites. In the second generation the bond is replaced by a set of four bonds, each of which

(*) Permanent address: Physics Department, Carnegie Mellon University, Pittsburgh, PA 15123,

${ }^{(* *)}$ Laboratoire de l'Institut de Recherche Fondamentale du Commissariat à l'Energie Atomique. 


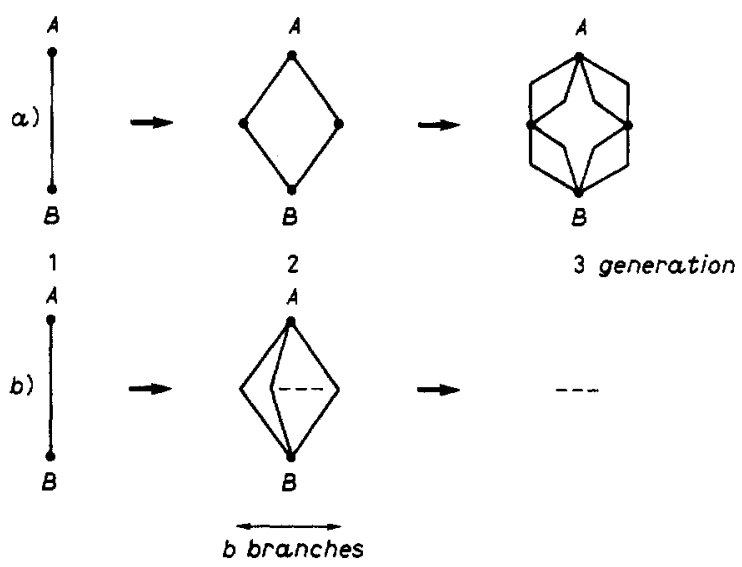

Fig. 1. - The iterative construction of the diamond hierarchical lattice (a)) and of the generalized lattice $(b)$ ).

is replaced by a corresponding set to form the third generation, etc. In the generalization shown in fig. $1 b$ ), each bond is replaced by a set of $2 b$ bonds. We shall consider directed walks from $A$ to $B$, that is paths of (minimal) length $L=2^{n-1}$ in a lattice of generation $n$.

To each bond of the lattice is assigned a random energy $e_{1}$ with probability $P_{1}\left(e_{1}\right)$. The energy $E_{W}$ of a directed walk $W$ from $A$ to $B$ is the sum of the energies of the bonds in the walk, and the ground-state energy $E$ is the minimum of $E_{W}$ over all $W$. The hierarchical nature of the lattice means that the ground-state energy $E^{(n+1)}$ of a lattice of generation $(n+1)$ can be written as

$$
E^{(n+1)}=\min \left(E_{1}^{(n)}+E_{2}^{(n)}, E_{3}^{(n)}+E_{4}^{(n)}, \ldots, E_{2 b-1}^{(n)}+E_{2 b}^{(n)}\right),
$$

where the $E_{i}^{(n)}$ are the ground-state energies of lattices of generation $n$. As these are independent random variables, (1) yields a recursion relation for the probability distribution $P_{n}\left(E^{(n)}\right)$ :

$$
\begin{aligned}
& Q_{n}(x)=\int \mathrm{d} y P_{n}(x-y) P_{n}(y), \\
& \int_{x}^{\infty} P_{n, 1}(y) \mathrm{d} y=\left[\int_{x}^{\infty} Q_{n}(y) \mathrm{d} y\right]^{b} .
\end{aligned}
$$

The first step (2) corresponds to the addition of two energies, and the second (3), to finding the minimum: note that the minimum of the $b$ sums in (1) is greater than $x$, if and only if each sum is greater than $x$.

As in the case of other disordered models on hierarchical lattices, one has to iterate a probability distribution. This usually cannot be done exactly and one has either to make approximations [12], to treat the problem numerically [13] or to expand around situations where the iteration of $P_{n}(x)$ is simple $[14,15]$. We used a numerical procedure for general $b$ and an expansion around $b=1$. 
Our numerical analysis was applied to distributions of the form

$$
P_{n}(x)=\sum_{m} a_{n}(m) \delta(x-m)
$$

where the sum is over a finite set of integers, because the recursion formula for the $a_{n}(m)$ is simple and easy to program. In the case

$$
a_{1}(-1)=1-p, \quad a_{1}(1)=p,
$$

we found that $P_{n}(x)$ tends toward the form

$$
P_{n}(x)=\delta_{n}^{-1} F_{b}\left[\left(x-\gamma_{n}\right) / \delta_{n}\right]
$$

with increasing $n$, provided $p$ is large enough, see (5), so that the -1 bonds do not percolate between $A$ and $B$. Here $\gamma_{n}$ and $\delta_{n}$ depend on the initial distribution, but $F_{b}$ depends only on $b$. The same form arose for an initial distribution with the $a_{1}(m)$ chosen to approximate a Gaussian. We also calculated $\mu_{n}(j)$, the $j$-th moment of $P_{n}(x)$ about the mean, $\left\langle(x-\bar{x})^{j}\right\rangle$, for several values of $j$ and found that ratios like $\mu_{n}(4) /\left[\mu_{n}(2)\right]^{2}$ become independent of $n$ as $n$ increases. Also the square root of $\mu_{n+1}(2) / \mu_{n}(2)$ tends to a limit

$$
\lambda=2^{\omega}=\lim _{n \rightarrow \infty}\left(\hat{\delta}_{n+1} / \hat{\delta}_{n}\right)
$$

which depends on $b$ but not on $P_{1}(x)$, see fig. $2 a$ ). Our numerical estimates for $\bar{e}$, the average ground-state energy per unit length of the walk as the latter becomes infinite, are shown in fig. $2 b$ ) for the initial distribution (5) with $p=1 / 2$, and for a set of values $a_{1}(m)$ chosen to approximate a Gaussian with mean 0 and variance 1 . For large $b, \bar{e}$ depends on $P_{1}(x)$, but for small $b$ both these distributions (which have the same mean and variance) give similar results.
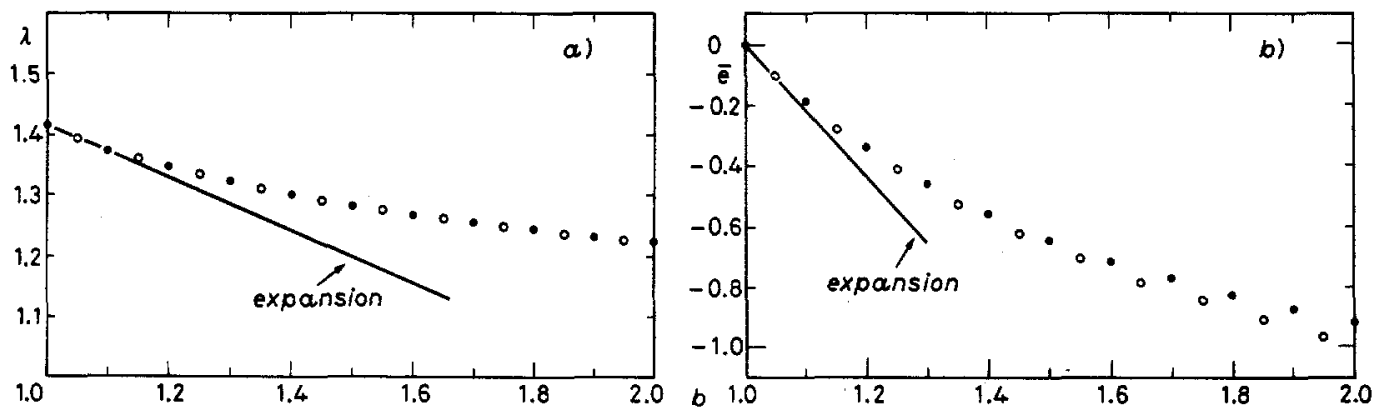

Fig. 2. - The scaling factor $\lambda(a)$ ) and the ground-state energy $\bar{e}(b))$ as a function of $b$ for initial conditions (7) and (13). delta functions, O Gaussian.

The fluctuations $\left\langle(E-\bar{E})^{2}\right\rangle$ of the ground-state energy vary with the length $L$ of the walk as $L^{2 \omega}$ when $L$ is large, because with each iteration $L$ doubles and the width of $P_{n}$ is multiplied by $2^{\omega}$, see (7). In the case $b=2$, which corresponds to the Migdal-Kadanoff approximation of the $2 d$ lattice, our numerical method gives $\lambda \simeq 1.23$ (i.e. $\omega \simeq 0.30$ ), whereas the exact value is known to be $\omega=1 / 3$ in two dimensions [6]. 
In the limit $b=1, P_{n+1}$ is the same as $Q_{n}$, and thus, for large $n, P_{n}$ will tend to a Gaussian distribution, assuming $P_{1}$ has finite moments, and one has $\lambda=\sqrt{2}$ and $e=0$ (assuming the first moment of $P_{1}$ vanishes), consistent with fig. 2 .

For $b$ close to 1 , we have carried out an analytic expansion to first order in $\varepsilon=b-1$ which confirms the numerical results. Assume that $P_{n}$ is a Gaussian with a small correction

$$
P_{n}(x)=\frac{1}{\sqrt{2 \pi} \delta_{n}} \exp \left[-\frac{1}{2}\left(\frac{x-\gamma_{n}}{\delta_{n}}\right)^{2}\right]\left\{1+\varepsilon \varphi_{n}\left(\frac{x-\gamma_{n}}{\delta_{n}}\right)+O\left(\varepsilon^{2}\right)\right\} \text {. }
$$

For $P_{n}$ to be normalized, $\varphi_{n}$ has to satisfy

$$
\int \mathrm{d} z \varphi_{n}(z) \exp \left[-z^{2} / 2\right]=0 .
$$

By means of (2) and (3) and ignoring terms of order $\varepsilon^{2}=(b-1)^{2}$, one obtains the expression

$$
P_{n+1}(x)=\frac{1}{2 \sqrt{\pi \hat{\partial}_{n}}} \exp \left[-\frac{1}{4}\left(\frac{x-2 \gamma_{n}}{\delta_{n}}\right)^{2}\right]\left\{1+\varepsilon+\varepsilon F\left(\frac{x-2 \gamma_{n}}{\delta_{n} \sqrt{2}}\right)+\varepsilon \mathscr{L} \phi_{n}\left(\frac{x-2 \gamma_{n}}{\delta_{n} \sqrt{2}}\right)\right\}
$$

where $F$ and the linear operator $\mathscr{L}_{\varphi}$ are defined by

$$
\begin{gathered}
F(z)=\log \left[(2 \pi)^{-1 / 2} \int_{z}^{\infty} \mathrm{d} t \exp \left[-t^{2} / 2\right],\right. \\
\mathscr{L}_{\varphi}(z)=(2 / \pi)^{1 / 2} \int \mathrm{d} t \varphi(t / \sqrt{2}) \exp \left[-(t-z)^{2} / 2\right] .
\end{gathered}
$$

In order to rewrite (10) in the form (8), we introduce two arbitrary constants $c_{n}$ and $d_{n}$ in the expressions

$$
\gamma_{n+1}=2 \gamma_{n}+\varepsilon c_{n} \sqrt{2} \delta_{n}, \quad \delta_{n+1}=\left(1+\varepsilon d_{n}\right) \sqrt{2} \delta_{n} .
$$

Then $\Phi_{n+1}$ is of the form

$$
\varphi_{n+1}(z)=a_{n}-c_{n} z+d_{n}\left(1-z^{2}\right)+F(z)+\mathscr{L} \varphi_{n}(z)
$$

with $a_{n}=1$ (see eq. (10)).

The linear operator $\mathscr{L}$ in (12) has eigenvalues $2^{1-s / 2}, S=0,1,2, \ldots$, associated with eigenfunctions (Hermite polynomials)

$$
\phi^{(S)}(z)=(-1)^{s}\left(\exp \left[z^{2} / 2\right]\right)(\mathrm{d} / \mathrm{d} z)^{s}\left(\exp \left[-z^{2} / 2\right]\right) .
$$

Thus the first three terms on the right side of (14) are proportional to $\varphi^{(0)}, \varphi^{(1)}$ and $\phi^{(2)}$, respectively.

If $F(z)$ is expanded as a series in the eigenfunctions (15), the $S=0$ (constant) term is cancelled by $a_{n}$. Next $c_{n}$ and $d_{n}$ can be chosen to cancel the $S=1$ and $S=2$ terms, respectively. Then, if the corresponding expansion for $\varphi_{n}$ contains only terms with $S \geqslant 3$, the same will be true of $\varphi_{n+1}$, and the fact that the corresponding eigenvalues are all less than 1 in magnitude means that $\phi_{n}$ will converge to a unique limit as $n \rightarrow \infty$. These considerations 
single out the unique values

$$
\left\{\begin{array}{l}
c_{n}=-K_{1}=(2 \pi)^{-1 / 2} \int \mathrm{d} z z F(z) \exp \left[-z^{2} / 2\right] \\
d_{n}=K_{2}=(2 \pi)^{-1 / 2} \int \mathrm{d} z \frac{1}{2}\left(1-z^{2}\right) F(z) \exp \left[-z^{2} / 2\right]
\end{array}\right.
$$

with numerical values $K_{1}=0.903197 \ldots, K_{2}=0.29782 \ldots$.

Upon iterating (13) using (16), and $\gamma_{1}=0, \delta_{1}=1$ (Gaussian of zero mean and of width 1), one obtains

$$
\begin{gathered}
\delta_{n}=\left[\sqrt{2}\left(1-\varepsilon K_{2}\right)\right]^{n-1}, \quad \gamma_{n} / 2^{n-1}=-K_{1} \varepsilon\left[1-2^{-(n-1) / 2}\right] /(\sqrt{2}-1), \\
\lambda=2^{\omega}=\sqrt{2}\left(1-\varepsilon K_{2}\right), \quad \tilde{e}=-K_{1} \varepsilon /(\sqrt{2}-1)
\end{gathered}
$$

to lowest order in $\varepsilon$; note the $\bar{e}$ is the large $n$ limit of $\gamma_{n} / 2^{n-1}$. The terms linear in $\varepsilon$ are shown as straight lines in fig. 2 , in good agreement with numerical results.

It would be of interest to extend the results described above in various ways. An obvious possibility is to consider alternative hierarchical lattices, such as one in which each of the $b$ branches in fig. $1 b$ ) contains a bond with $a>2$. More challenging is the problem at finite temperature where each walk is given a weight $\exp \left[-E_{W} / T\right]$, and (1) is replaced by a corresponding recursion formula for the partition function $Z^{(n)}$. If $(\log Z)$ could be obtained as an expansion in powers of $b-1$, in analogy with the ground state, this would provide a new class of almost solvable random systems. One could then for example, see if the replica method with an appropriate breaking of symmetry yields the same result, as in the case in the "mean-field" situation of a polymer of a tree $[9,16]$. Finally we note that if the initial distribution $P_{1}(x)$ of ground-state energies decays sufficiently slowly as $|x| \rightarrow \infty$, the limiting distribution for large $n$ and $b=1$ is not a Gaussian, but a Levy distribution [17]. It would be of interest to see if our expansion around $b=1$ could be extended to that case.

$$
* * *
$$

We would like to thank P. Collet, C. M. Newman and H. SPoHN for useful discussions. BD thanks the IBM Bergen Scientific Centre (Norway) where part of this work was done. RBG gratefully acknowledges the hospitality of the C.E.N. Saclay, and financial support from the U.S. National Science Foundation Grant DMR 8613218.

\section{REFERENCES}

[1] Chakrabarti B. K. and Kertosz J., Z. Phys. B, 44 (1981) 221.

[2] Kremer K., Z. Phys. B, 45 (1981) 149.

[3] Derrida B., J. Phys. A, 15 (1982) L-119.

[4] Harris A. B., Z. Phys. B, 49 (1983) 347.

[5] Zhang Y. C., Phys. Rev. Lett., 59 (1987) 2125.

[6] Kardar M. and Zhang Y. C., Phys. Rev. Lett., 58 (1987) 2087.

[7] Huse D. A., Henley C. L. and Fisher D. S., Phys. Rev. Lett., 55 (1985) 2924.

[8] VON BeiJeren H., KUTNER R. and SPOHN H., Phys. Rev. Lett., 54 (1985) 2026.

[9] DerridA B. and SpOHN H., J. Stat. Phys., 51 (1988) 817. 
[10] Berker A. N. and Ostlund S., J. Phys. C, 12 (1979) 4961.

[11] Kaufman M. and Griffiths R. B., Phys. Rev. B, 24 (1981) 496; 30 (1984) 244.

[12] Kinzel W. and Domany E., Phys. Rev. B, 23 (1981) 3421.

[13] Andelman D. and Berker A. N., Phys. Rev. B, 29 (1984) 2630.

[14] Derrida B. and Gardner E., J. Phys. A, 17 (1984) 3223.

[15] Gardner E., J. Phys. (Paris), 45 (1984) 1755.

[16] Derrida B. and Gardner E., J. Phys. C, 19 (1986) 2253, 5783.

[17] Montroll E. W. and Bendler J. T., J. Stat. Phys., 34 (1984) 129. 